\title{
Relationship of Serum Leptin, Lactate Dehydrogenase Levels and Severity in Preeclampsia
}

\author{
Uma Devi Kantipudi ${ }^{1}$, SR Sheela ${ }^{1},{ }^{*}$ CD Dayanand ${ }^{2}$, Nagarjuna Sivaraj ${ }^{3}$ \\ ${ }_{2}^{1}$ Department of OBG Sri Devaraj Urs Medical College Kolar, Karnataka, India. \\ $*^{2}$ \\ $*$ \\ Department of Biochemistry Sri Devaraj Urs Medical College, Kolar, Karnataka, \\ India. \\ 3 Department of Cell Biology and Molecular Genetics, Kolar, Karnataka, India. \\ Email:cd8905@yahoo.co.in
}

\begin{abstract}
Aim: To assess whether Serum Leptin and Lactate dehydrogenase levels as an indication of severity in preeclampsia. Study Design: A prospective case control study, consist of two groups such as group 1 normotensive Pregnants and group 2 as cases with clinically diagnosed preeclampsia Place and Duration of Study: Department of Obstetrics and Gynaecology, RL Jalappa Hospital and Research Centre and Proteomics laboratory kolar, between January 2013 and July 2014. Methodology: A total number of 100 pregnant patients were enrolled in the present study. Amongst, normotensive and preeclamptic pregnant women Group $1(n=50)$ as controls $(n=50)$. Group-2 $(n=50)$ were preeclampsia cases. Five $\mathrm{ml}$ of blood samples were collected from each normal pregnant and preeclampsia patients. Leptin levels and lactate dehydrogenase parameters were estimated using ELISA -Micro plate Reader method. Statistical analysis analysed by using SPSS Software. Results: The Mean \pm SD values of Lactate dehydrogenase IU/L (399.04 \pm 113.08$)$ and Leptinng $/ \mathrm{ml}(9.02 \pm 4.65)$ in normal pregnant and Lactate dehydrogenase IU/L (1296.68 \pm 1732.95$)$, Leptin $\mathrm{ng} / \mathrm{ml}(23.32 \pm 8.78)$ in preeclampsia cases were presented. Similarly Mean $\pm S D$ values in preeclampsia were presented respectively. Conclusion: The elevated serum leptin levels in preeclampsia indicate endothelial dysfunction involved in the pathogenesis of preeclampsia. The relationship of serum leptin and Lactate dehydrogenase levels were increased in preeclampsia that is directly proportional to gestational age in last trimester. These biochemical parameters were significantly elevated in severe preeclampsia, mild preeclampsia and compared to normal pregnancy. Identification of high-risk patients with elevated levels of serum lactate dehydrogenase and Leptin necessitate the close monitoring for prompt and correct management which may decrease the complications of disease condition and also facilitate to reduce maternal and fetal morbidity and mortality.
\end{abstract}

Keywords: Preeclampsia; Leptin; Normotensive; Lactate Dehydrogenase

\section{Introduction}

Preeclampsia is a clinical condition characterized by hypertension and proteinuria. This obstetric complications leading to intrauterine growth restriction, preterm delivery, maternal and fetal morbidity and mortality. The reversible changes generally occurs in preeclampsia after delivery. It occurs in 5-7 \% of the pregnancies worldwide. The incidence is still higher in India of around $8-10 \% .{ }^{4}$ As per the World Health Report the maternal mortality during pregnancy and puerperium is around $12 \%$. In developing countries, $17 \%$ of direct obstetric deaths are as a result of hypertension. The mortality rate of preeclampsia in the developing and developed countries varies approximately eight hundred women die from pregnancy and child birth related complications around the world every day.

The disorder is probably multifactorial, although most cases of preeclampsia are 
South American Journal of Academic Research

Special Issue May 2016

characterized by abnormal maternal uterine vascular remodeling by fetally derived placental trophoblast cells. Reports are available on animal models that have been used to study various aspects of preeclampsia, amongst the common model is of placental oxygen dysregulation, abnormal trophoblast invasion, inappropriate maternal vascular damage and anomalous maternal-fetal immune interactions. Preeclampsia more commonly occurs in first pregnancies then subsequent pregnancies. Potential causes and mechanisms behind preeclampsia remain unknown, but maternal immune, genetic factors and placenta have been implicated. There are convincing evidences that indicates the obesity increases the risk of preeclampsia. Obesity is major disease condition observed commonly in developed countries. The relationship of obesity with respect to type2 diabetes mellitus and cardiovascular disease are studied. However obesity is also having important implications for pregnancy outcomes, relating to these preeclampsia adverse outcomes are linked with obesity.

Leptin is a polypeptide hormone translated from Obesity gene located on chromosome 7. The molecular weight of leptin is 16-KD Containing 146 amino acid residues which is released from the adipocytes and placental syncytiotrophoblasts and exerts its action on binding to leptin receptor in hypothalamus to regulate energy homeostasis. Leptin receptor is abundantly expressed in various maternal tissues, placenta and fetal tissues, so physiological and pathophysiological roles of leptin in pregnancy are expected. However, the role of leptin in pregnancy has not been fully elucidated yet.

Lactate dehydrogenase (LDH-EC 1.1.1.27) is an Intracellular enzyme most often measured to evaluate the extent of Cellular death due to its wide distribution in heart, liver, kidney, skeletal muscle, brain, lungs and blood cells. In Acute preeclampsia it leads to fetal mortality and morbidity with increased activity of AST and LDH.

The aim of the present study focused on evaluating the Serum leptin level and LDH level in preeclampsia in comparison with the normal pregnancy to understand that can serve as a marker to assess the severity of Preeclampsia and also to plan a strategy to improve the Maternal and Fetal outcome.

\section{Materials and Methods}

\subsection{Source of Data}

\subsubsection{Patients}

A total number of 100 subjects were enrolled in the present study. Amongst, Group1 $(n=50)$ normotensive pregnant women as controls. Group-2 $(n=50)$ were preeclampsia cases. These patients were clinically diagnosed by OBG dept. of RL Jalappa Hospital and Research Centre between "January 2013 to July 2014" and were participating in the study. After obtaining approval by the Institutional ethics committee and also informed patient consent the study was carried out. A standard Sri Devaraj Urs University Proforma was used to collect the data. Five $\mathrm{ml}$ of blood samples were collected in to a plain vacutainer and stored at $-80^{\circ} \mathrm{C}$ until analysis. LDH and Leptin parameters were determined using Dry Chemistry analyzer and Sand witch ELISA method respectively.

Pregnants beyond 20 weeks of gestation were clinically diagnosed with preeclampsia as per National High Blood Pressure Education Programme working group (NHBPEP) Classification with blood pressure $\geq 140 / 90 \mathrm{~mm}$ of $\mathrm{Hg}$ and with proteinuria were included in the study group as cases. Age, gestation, and Parity matched normotensive, Non preeclamptic pregnants were also included in the study as controls.

Patients with pre-existing thyroid disease, history of renal disease and history of any metabolic disorder before or during the pregnancy, history of chronic hypertension and history of medication known to affect the thyroid function are excluded from the study.

\subsection{Methods}

Preeclampsia diagnosed as blood pressure of $\geq 140 / 90 \mathrm{~mm}$ of $\mathrm{Hg}$ noted for the first time during pregnancy on $\geq 2$ occasions at least 6 hours apart, after 20 weeks of gestation with 
proteinuria of $\geq 300 \mathrm{mg} / 24$ hours or $\geq 1+$ by a dipstick method in a random urine sample (NHBPEP and ACOG criteria)

\subsection{Estimation of serum leptin and LDH level.}

The DRG Leptin ELISA Kit is a solid phase enzyme linked immunosorbent assay (ELISA) based on the sandwich principle. The microtitre wells were coated with a monoclonal antibody directed towards a unique antigenic site on a Leptin molecule. An aliquot of patient sample containing endogenous leptin was incubated in the coated well with a specific rabbit anti Leptin antibody. A sandwich complex was formed after incubation, the unbound material was washed off and an anti-rabbit peroxidase conjugate was added for detection of the bound Leptin with the substrate solution. The intensity of color developed was proportional to the concentration of Leptin in the patient sample.

LDH is measured by Dry chemistry analyzer (Johnson and Johnson vitros 250)

\subsection{Statistical Analysis}

The results obtained were analyzed by using statistical tools such as Mean, SD and results on categorical variables are presented as Numbers and Percentage (\%). 't' test was used to find the significance of study parameters between two groups. The level of significance between the groups were presented with $\mathrm{P}$ value: $0.005<\mathrm{P}<0.10$, moderately significance with $\mathrm{P}$ value: $0.01<\mathrm{P} \leq 0.05$, and Strongly significant with $\mathrm{P}$ value $\mathrm{P} \leq 0.01$.

\section{Results}

The results obtained from the study on age group, gravid type, gestational age group distribution, blood pressure were presented in the tabular format in Table, showing details of age group, gravid, gestational age, and blood pressure between normotensive and preeclampsia group.

Accordingly, amongst the patients who visited the hospital, majority of the age group falls in the range of 18 to 30 years. The Data analysis indicated from the study group, particularly in Normotensive group, $58 \%$ were primigravida and $42 \%$ were multigravida where as in the Preeclampsia group, $66 \%$ were primigravida and $34 \%$ were multigravida observed as shown. Similarly, regarding gestational age group distribution, $80 \%$ of the normotensive patients were in the gestational age group of 33 to 40 weeks while $80 \%$ of the preeclampsia patients were in this group. The mean Systolic Blood pressure in normotensive group was 118.00 \pm 7.28 and in preeclampsia group was $154.00 \pm 13.85$ as mean diastolic blood pressure

Syndrome of hypertension, with proteinuria and/or edema. In majority of the patients, the clinical presentation is mild, only with a slight increase in blood pressure or proteins in the urine. Severe maternal and fetal complications such as the HELP syndrome, eclampsia, preterm delivery, abruption placenta, intrauterine fetal death or fetal growth restriction are seen in a minority of patients.

Table 1. Characteristics of the normal pregnant and preeclamptic women

\begin{tabular}{llllll}
\hline Particulars & \multicolumn{2}{l}{ Normotensive $\mathbf{( N = 5 0 )}$} & \multicolumn{2}{l}{ Preeclampsia (N=50) } \\
\cline { 2 - 6 } & Age group & Numbers & Percentage & Numbers & Percentage \\
\hline Age in years & $18-20$ & 12 & 24 & 12 & 24 \\
& $21-25$ & 25 & 50 & 25 & 50 \\
& $26-30$ & 10 & 20 & 10 & 20 \\
& $>30$ & 03 & 06 & 03 & 06 \\
\hline Gestation age in weeks & Weeks & Numbers & Percentage & Numbers & Percentage \\
& $28-32$ & 05 & 10 & 05 & 10 \\
& $33-36$ & 14 & 28 & 14 & 28 \\
& $37-40$ & 30 & 60 & 30 & 60 \\
\hline & $>40$ & 01 & 02 & 01 & 02 \\
\hline Parity distribution & Parity type & Numbers & Percentage & Numbers & Percentage \\
\hline & Primi gravid & 29 & 58 & 33 & 66
\end{tabular}


South American Journal of Academic Research

Special Issue May 2016

\begin{tabular}{|c|c|c|c|}
\hline & Multigravida & 42 & 17 \\
\hline & Types & Mean, SD value & Mean, SD value \\
\hline Blood pressure in $\mathrm{mm} \mathrm{Hg}$ & $\begin{array}{l}\text { Systolic BP } \\
\text { Diastolic BP }\end{array}$ & $\begin{array}{l}118.00 \pm 7.28 \\
75.60 \pm 5.77\end{array}$ & $\begin{array}{l}154.00 \pm 13.85 \\
101.40 \pm 10.50\end{array}$ \\
\hline
\end{tabular}

Maternal age is one of the essential risk factors in women with Preeclampsia. The risk of pre-eclampsia is higher when the age of pregnant women is less than 25 years[11] In the present study, the majority of the patients selected in both the groups were in the age group of 21-25 years, which comprising $50 \%$ in the control group and $50 \%$ in the preeclampsia group. However, this study shown blood pressure variation in the normotensive group was $75.60 \pm$ 5.77 and in preeclampsia group was $101.40 \pm 10.50$. As per the results of Leptin parameter, the mean value of preeclampsia group (23.32 \pm 8.78$)$ is significantly higher and the mean value of normotensive group $(9.02 \pm 4.65)$ and the significant $P$ value is $(p=<0.001)$ which is shown in table, Comparison of Thyroid hormone levels between control and preeclampsia groups.

As per the results of Lactate dehydrogenase parameters, the mean preeclampsia group levels are significantly elevated in the study group as compared to the control group. $(p<0.001)$ whereas the mean Leptin and LDH values are comparable in the two groups. Leptin and LDH ratio clearly indicating the atherogenic potential in the preeclampsia groups table, Comparison of control and preeclampsia groups.

Table 1. Comparison of leptin and Lactate dehydrogenaselevels between normotensive pregnant and preeclamptic groups

\begin{tabular}{|l|l|l|l|}
\hline Parameter & $\begin{array}{l}\text { Normotensive } \\
\text { group (Mean } \pm \\
\text { SD) }\end{array}$ & $\begin{array}{l}\text { Pre-eclampsia group } \\
\text { (Mean } \pm \text { SD) }\end{array}$ & p value \\
\hline Leptin & $9.02 \pm 4.65$ & $23.32 \pm 8.78$ & $<0.001 * *$ \\
\hline LDH(IU /L) & $399.04 \pm 113.08$ & $1296.68 \pm 1732.95$ & $<0.001 * *$ \\
\hline
\end{tabular}

Table 2. Comparison of leptinand LDH Levels between Mild and Severe preeclampsia

\begin{tabular}{|l|l|l|l|l|}
\hline Parameter & \pm SD & Mild & Severe & p Value \\
\hline Leptin(ng/ml $)$ & $23.32+8.78$ & $22.12+9.39$ & $35+8.58$ & $<0.001^{* *}$ \\
\hline $\mathrm{LDH}(\mathrm{IU} / \mathrm{L})$ & $1296.68+1732.95$ & $732.06+299.46$ & $1538.65+2022.41$ & $<0.001$ \\
\hline
\end{tabular}

\section{Discussion}

Preeclampsia is a hypertensive disorder of pregnancy caused by placental hypoxemia secondary to shallow endovascular cytotrophoblast invasion of spiral arteries. It has been regarded as one of the major causes of maternal morbidity and mortality. The frequency of occurrence of this condition is about 5-10\% of all pregnant women.(young et al., 2010). Preeclampsia is associated with multi organ dysfunction, that occurs due to vascular endothelial damage of maternal liver, kidney, lungs and nervous system. Blood, coagulation system and cellular dysfunction generally release excessive LDH leakage as observed in preeclampsia.

In majority of the patients, the clinical presentation is severe with much increase in blood pressure and proteins in urine and associated with maternal and fetal complications such as HELLP syndrome, eclampsia, preterm delivery, abruption placenta, still born, intrauterine growth restriction etc. Maternal age is one of the essential risk factors in women with preeclampsia. The risk of preeclampsia is higher when the age of pregnant women is less than 25 years. Even in our study the majority of the patients selected were in the age group of 21-25 yrs $(50 \%)$ in both the groups, followed by $18-20$ yrs $(24 \%)$. The calculated mean age among the normotensive group was $23.96 \pm 3.88$ and the mean age among the preeclampsia was $23.78 \pm 3.64$. In a study of Kafulafula and his coworkers found that maternal age, parity and gestational age didn't show any correlation with serum leptin levels. However in our study the indicator gestational age is matching with elevated leptin level.

Several studies reported that women with preeclampsia are two times expected to be primiparous as women without hypertensive disorder of pregnancy. ${ }^{15-20}$ In our study $66 \%$ were 
primigravidae among the preeclampsia group where as $58 \%$ were primigravidae among controls. As per observation of our study, majority of the patients were in the gestational age group of 38-40 weeks belong to $60 \%$ in both the groups. The mean Leptin and LDH value is significantly higher in preeclampsia group as compared to normotensive group. (P value $<0.001)$. Firstly, this study revealed that serum leptin levels increased significantly during normal pregnancy. High leptin level may be increased in women with increased gestational age because not only adipose tissue is a source of leptin, but also in pregnancy fetus, placenta, amniotic fluid, increase in plasma volume and extra vascular fluid accumulation leads to increase in maternal weight which is responsible for increase in serum leptin level.

Our results are in line with those of Michael et al. (2004) who confirmed that leptin levels in preeclampsia are higher than normal pregnant as control group and with those of Singh et al. (2005) who found that leptin concentrations are significantly raised in fetoplacental tissues from women with preeclampsia. Recently, Sabiha et al. (2005) showed that serum leptin levels were significantly higher in pregnant women with preeclampsia.

Conversely, Kafulafula et al. (2002) and Salomon et al. (2003) did not find any difference in serum leptin levels between normotensive and preeclamptic pregnant women and others reported that leptin concentrations were significantly lower in women with preeclampsia than in normal pregnant women (Laml et al., 2001). ${ }^{23}$

On the other hand, serum leptin levels showed a significant positive correlation with systolic, diastolic blood pressure and proteinuria in both mild and severe preeclamptic groups. Secondly, our study indicated that serum leptin levels increase markedly in severe preeclampsia. There was a significant increase in serum leptin levels in severe preeclamptic group in comparison with normal pregnant (3rd trimester) and mild preeclamptic groups matched for gestational age.

The results of our study are supported by those of Mise et al. (1998) who observed that plasma leptin levels were elevated significantly in pregnant women with PE. Furthermore, plasma leptin levels in the severe PE group were significantly higher than those in the mild preeclampsia group.

The increase in serum leptin levels in preeclampsia may be a response to, or a result of an inappropriate trophoblastic invasion of spiral arteries (Sebiha et al., 2005).

Leptin is a type 1 cytokine and its expression may be up-regulated during placentation and afterwards. It has been recently reported that the level of maternal serum leptin was increased and correlated positively with the level of TNF- $\alpha$ and IL-6 in preeclampsia (Bartha et al., 2001).

The results of our study showed that lactate dehydrogenase levels were significantly higher in women with severe preeclampsia when compared with mild preeclampsia which is statistically significant. $(\mathrm{P}=<0.001)$. The elevated $\mathrm{LDH}$ levels in preeclampsia are also found in many other studies. Lactate dehydrogenase is an intracellular enzyme that converts lactic acid into pyruvicacid and elevated levels indicate cellular death and leakage of enzyme from the cell as shown in our study and also were supported by HS Qublan 2005.

LDH may be increased due to liver damage. This endothelial vascular damage is the main cause in the occurrence of preeclampsia.

Qublan et al found in their study that LDH levels were significantly elevated in women with preeclampsia and eclampsia $(<0.001)$. Higher LDH levels had significant correlation with high blood pressure $(P<0.10)$ as well as poor maternal and perinatal outcome. High serum LDH levels correlate well with the severity of the disease and poor outcomes in patients of preeclampsia and eclampsia. The mean gestational age at the time of delivery in his study was significantly less in patients with increasing LDH levels $(\mathrm{P}=0.025)$. This indicates increase in preterm deliveries in patients with higher LDH levels. The association of Low birth weight of infants with increase in serum LDH levels was suggested by He et al. in their study. ${ }^{28}$ This was in contrary to Qublan et al. who did not find any significant association. In the present study it 
South American Journal of Academic Research

Special Issue May 2016

was observed that there was significant association of low birth weight and increasing LDH levels $(\mathrm{P}=0.019)$. This could partially be due to higher incidence of premature births in this group.

Qublan HS in his study showed $92 \%$ patients had multi-organ dysfunction in severe preeclamptic women having LDH>800 IU/1. Severely preeclamptic women with LDH $>800$ IU/1 showed significant increase in terms of eclampsia, abruptio placenta, intracranial hemorrhage, HELLP syndrome, acute renal failure, disseminated intravascular coagulation, and pulmonary edema compared with women who had lower levels $(\mathrm{p}<0.001)$. A high serum level of $\operatorname{LDH}(>1,400 \mathrm{IU} / \mathrm{L})$ were shown to have a high predictive value for significant maternal morbidity in a study conducted by Martin et al. ${ }^{90}$ Demier et al. concluded that there was a statistically significant relation between maternal complications and high LDH levels. It was noted that in early onset severe Preeclampsia, LDH levels before delivery were significantly higher in the abruption group Odendaal et al.

Therefore, Measurement of serum Leptin and LDH in normal pregnant, mild preeclampsia and severe preeclampsia has become appropriate to understand and assess fetal morbidity.

\section{Conclusion}

The serum leptin levels are higher in preeclampsia in comparison to normotensive pregnant women that might contribute to endothelial dysfunction involved in the pathogenesis of preeclampsia. The serum leptin and Lactate dehydrogenase were increased in preeclampsia that is directly proportional to gestational age at last trimester. These two biochemical parameters were significantly elevated in severe preeclampsia condition than compared with mild preeclampsia and normal pregnancy clearly indicated to understand the extent of severity and thus can be considered as useful biomarker. Identification of high-risk patients with elevated levels of serum lactate dehydrogenase and Leptin necessitate the close monitoring for prompt and correct management which may decrease the complications of disease condition and also facilitate to reduce maternal and fetal morbidity and mortality.

\section{Competing Interests}

Authors have declared that no competing interests exist.

\section{Acknowledgements:}

We would like to thank the authorities of Sri Devaraj Urs Academy of Higher Education and Research for supporting this study.

\section{References}

[1.] Cindrova-Davies T. Gabor Than award lecture 2008: preeclampsia-From placental Oxidative stress to maternal endothelial dysfunction. Placenta 2009; 30:55-65.

[2.] Von Dadleszen, P. Mageela, Taylor, Muir J C, Stewart SD, et al. Maternal hypertension and neonatal outcome among small for gestational age infants. Obstet Gynecol 2005; 106:335-39.

[3.] Cunningham F G, Leveno KJ, Bloom SL, Hauth J C, Gilstrap D J, Wenstrom S Y. Williams Obstetrics. 23rd Edition. McGraw Hill Medical Publishing Division 2010; section VII, 34: 706-56.

[4.] Mutlu T U, Ademoglu E. Imbalance between lipid peroxidation, antioxidant status in preeclampsia. Gynecol Obstet Invest 1998; 46: 37-40.

[5.] Maternal mortality in 2005: estimates developed by WHO, UNICEF, UNIFPA and the World Bank, Geneva, World Health Organization, 2007.

[6.] World Health Organization Fact Sheet, May 2012.

[7.] Kathleen A. Pennington, Jessica M. Schlitt, Daniel L .Jackson, Laura C. Schulz and Danny J. Schust: Preeclampsia: Multiple approaches for a multifactorial disease, disease models and mechanisms 5, 918(2012).

[8.] Misra A, Khurana L. Obesity and the metabolic syndrome in developing countries. J. of Clin Endo and Metabolism

[9.] Ursula M, Axel M. Gressner: Endocrine regulation of energy metabolism, clinchemis 50:9 1511-1525 (2004). 
[10.] Malarewicz A, Gruszka O, Szymkiewicz J, RogalaJ. The usefulness of routine laboratory tests in the evaluation of sudden threat of pregnant woman and the fetus in preeclampsia. Ginekol Pol 2006.,77(4):27684.

[11.] Procopciuc LM, Hazi GM, Caracostea G: Correlation between the TSHRc Asp727Glu polymorphism and plasma thyroid stimulating hormone levels in Romanian preeclamptic women. Gynecol Endocrinol. 2011; 27(4): 225-231.

[12.] Naglaa G, Ashraf D, Rania A, and Alaa El H: "Evaluation of serum Leptin and Androgens Levels in Preeclampsia: Relation with Disease Severity”. J. American sci 2011. 7(9):366-372.

[13.] Rubina A, Tabassum M: "Relation between Preeclampsia and Cardiac Enzymes" J. ARYA Atherosclerosis 2008, 4(1): 29-32.

[14.] Yadav S, Yadav R, Saxena U. Hypertensive disorders of pregnancy and perinatal outcome. $J$. Obset. Gynecol. Ind. 1997; 17: 322-30

[15.] Coonrod D V, Hickok DE, Zhu K, Easterling T R, Daling JR. Risk factors for Pre-eclampsia in twin pregnancies: a population-based cohort study. Obstet Gynecol 1995; 85:645-50.

[16.] Eskenazi B, Fenster L, Sidney SA. Multivariate analysis of risk factors for Pre-eclampsia. J Am Med Assoc 1991; 266:23741.

[17.] Stone J L, Lockwood CJ, Berkowitz GS, Alvarez M, Lapinski R, Berkowitz RL. Risk factors for severe Pre-eclampsia. Obstet Gynecol 1994; 83:357-61.

[18.] Chen CL, Cheng Y, Wang PH, Juang CM, Chiu LM, Yang MJ, et al. Review of pre-eclampsia in Taiwan: a multi-institutional study. Zhonghua Yi Xue Za Zhi (Taipei) 2000; 63:869-75.

[19.] Odegard RA, Vatten L J, Nilsen S T, Salvesen K A, Austgulen R. Risk factors and clinical manifestations of pre-eclampsia. Br J Obstet Gynecol 2000; 107:1410-6.

[20.] Stamilio DM, Sehdev HM, Morgan MA, Propert K, Macones GA. Can antenatal clinical and biochemical markers predict the development of severe Pre-eclampsia? Am J Obstet Gynecol 2000; 182: 589-94.

[21.] Salvatores M, Gennarelli G, Menato G, Massobrio M, Leptin as a possible marker of augmented metabolic risk during pregnancy. Minerva Ginecol 2006; 58(1):1-10.

[22.] Singh, H.J.; Abu Bakar, A.; CheRomli, A. and Nila, A. (2005): Raised leptin concentrations in feto-placental tissues from women with preeclampsia. Hypertens Pregnancy; 24(2): 191-199.

[23.] Laml, T.; Preyer, O.; Hartmann, B.W.; Ruecklinger, E.; Soeregi, G. and Wagenbichler, P. (2001): Decreased maternal serum leptin in pregnancies complicated by preeclampsia. J Soc Gynecol Invest; 8(2): 89-93.

[24.] Mise, H.; Sagawa, N.; Matsumoto, T.; Yura, S.; Nanno, H.; Itoh, H.; Mori, T.; Masuzaki, H.; Hosoda, K.; Ogawa, Y. and Nakao, K. (1998): Augmented placental production of leptin in pre-eclampsia: possible involvement of placental hypoxia. J. of Clin Endo and Metabolism; 83: 3225-3229.

[25.] SebihaOzkan; Cemal Tamer Erel; Riza Madazli and Kilic Aydinli (2005): Serum leptin levels in hypertensive disorder of pregnancy. Eur J. of Obstet and Gynecol and Reprod Biol; 120: 158-163.

[26.] Bartha, J. L.; Romero-Carmona, R.; Escobar-Llompan, M. and Comino-Del-gado, R. (2001): The relationships between leptin and inflammatory cytokines in women with preeclampsia. BJOG; 108(12): 1272-6.

[27.] Qublan HS, Ammarin V, Bataineh O, Al Shraideh Z, Tahat Y, Awamleh I, et al. Lactic dehydrogenase as a biochemical marker of adverse pregnancy outcome in severe pre-eclampsia. Med Sci Monit 2005; 11(8): CR393-CR397.

[28.] He S, Bremme K, Kallner A, et al. Increased concentrations of lactate dehydrogenase in pregnancy with Preeclampsia; a predictor for birth of small for gestational age infants. Gynecol Obstet Invest. 1995; 39:234-8.

[29.] Martin J N Jr, May WL, Magann E F, et al. Early risk assessment of severe Preeclampsia: admission battery of symptoms and laboratory tests to predict likelihood of subsequent significant maternal morbidity. Am J Obstet Gynecol. 1999; 180:1407-14.

[30.] Demir SC, Evruke C, Ozgunen FT, et al. Factors that influence morbidity and mortality in severe preeclampsia, eclampsia and HELLP syndrome. Saudi Med J. 2006; 27:1015-18.

[31.] Hall DR, Odendaaal H J, Kirsten G F, et al. Expectant Management of early onset, severe preeclampsia perinatal outcome. BJOG. 2000; 107:1258-64. 
South American Journal of Academic Research

Special Issue May 2016

\section{Authors' contributions:}

This work was carried out in collaboration between all authors. Dr. S R. Sheela and Dr. C. D. Dayanand designed the study, wrote the protocol, and wrote the first draft of the manuscript. Dr. Uma Devi Kantipudi managed the literature searches, analyses of the study performed the spectroscopy analysis and Mr. Nagarjuna. Sivaraj managed the experimental process. All authors read and approved the final manuscript. 